Forthcoming in Philosophical Psychology

\title{
Representationalism and the Determinacy of Visual Content
}

Ben Bronner

\begin{abstract}
Determinacy is the claim that covert shifts in visual attention sometimes affect the determinacy of visual content. (Capital letters will distinguish the claim from the familiar word, "determinacy.") Representationalism is the claim that visual phenomenology supervenes on visual representational content. Both claims are popular among contemporary philosophers of mind, and DETERMINACY has been employed in defense of representationalism. I claim that existing arguments in favor of DETERMINACY are inconclusive. As a result, DETERMINACY-based arguments in support of representationalism are not strong ones.
\end{abstract}

The representational content of a mental state is the way that state represents the world to be. The phenomenology of a mental state is what being in that state is like for a subject. A major project in contemporary philosophy of mind is determining the relationship between the content and the phenomenology of conscious mental states. The project is most advanced with regard to vision. As I will use the term, representationalism is the claim that visual phenomenology supervenes on visual content - in other words, that there cannot be two conscious visual states that differ in phenomenology without an accompanying difference in what they report about the world. There are other versions of representationalism but this is the version I will consider. Understood in this way representationalism is a popular view, endorsed by Harman (1990), Dretske (1995), Byrne (2001), Tye (2002), and others. Representationalism has recently been challenged by several examples involving covert shifts in visual attention. Covert shifts in attention involve no movement of the eyes. Nickel (2007) considers 
covertly shifting attention between different subsets of the nine squares of a $3 \times 3$ grid. (See Figure 1.) Such a grid can be experienced in two different ways (among others). Fix your eyes on the middle of the center square. Then, without moving your eyes, attend to the four corner squares and the center square. You should be able to attend to this set of squares as a group. Next, shift attention away from the first group of squares and to the remaining four squares. Try shifting attention back and forth a few times, concentrating on each set of squares as a group. The shift in attention should affect phenomenology. It is natural to say that the attended squares are more salient or prominent than the unattended squares. The attended squares stand out. Nickel argues that such a shift in attention changes visual phenomenology but not visual content. If Nickel is correct, then representationalism is false. Chalmers (2004) considers a broadly similar example, although he allows that attention might affect visual content in such a case. Speaks (2010) offers another example of this type, arguing that representationalism is safe only if the phenomenology of attention does not count as visual phenomenology.

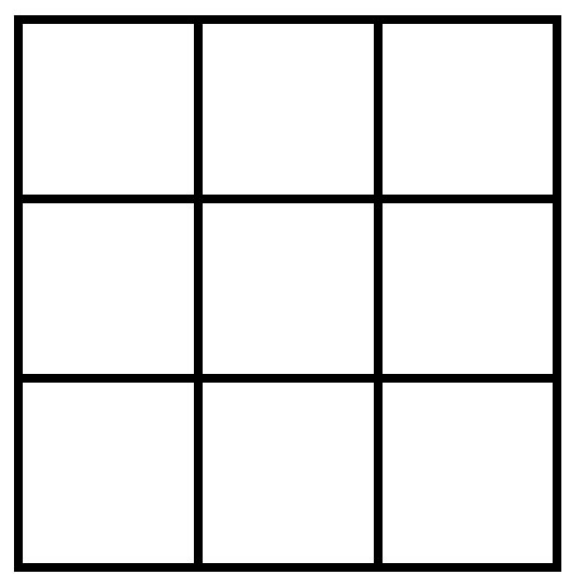

Figure 1. A 3x3 grid like that used by Nickel (2007). 
One response to this type of example appeals to the (alleged) fact that visual content comes in varying degrees of determinacy. The determinacy of visual content depends upon the determinacy of the properties that vision attributes to the scene before the eyes. Sometimes visual content may be relatively indeterminate, as when you see an object via peripheral vision. Perhaps your eyes tell you that such an object is red, but not which shade of red. Or perhaps that the object's facing surface is round, but not whether it is circular or elliptical. For our purposes, we can think of the determinacy of a property as its specificity along some dimension of variation, such as the dimensions of color, shape, size, and texture. Scarlet is more determinate than red because the former is a more specific color property than the latter.

Suppose that in the examples of Nickel, Chalmers, and Speaks, shifting attention alters the determinacy of visual content. In particular, suppose that vision attributes properties of greater determinacy to items that are attended. In that case representationalism would be safe. To falsify representationalism we need an example where visual phenomenology changes and yet visual content remains the same. And one way for visual content to change is for the determinacy of visual content to change. Nanay (2010) claims that attention always affects the determinacy of visual content. Ganson and I (2013) argue that attention sometimes affects the determinacy of visual content, that the examples in question might well involve such a change in content, and hence that they are not effective counterexamples to representationalism. In order for such responses to work, the following claim must be true.

(DETERMINACY) Covert shifts in visual attention sometimes affect the determinacy of visual content. 
In what follows, I change my position on the matter. I now claim that existing arguments in favor of DETERMINACY are inconclusive. As a result, DETERMINACY-based responses to the examples of Nickel, Chalmers, and Speaks are not strong ones. ${ }^{1}$

Several authors endorse DETERMINACY without appealing to empirical evidence. Chalmers says that in "typical cases" shifts in attention affect visual determinacy, "for example, shifting attention to a word may lead one to represent the shapes of its letters with greater specificity" (2004: 161). Nanay goes so far as to say that shifts in attention always affect visual determinacy: "[A] change in our attention changes the determinacy of the properties attributed to the perceived scene. In other words [...] perceptual content is always affected by the allocation of one's attention" (2010: 269-270). Both Chalmers and Nanay are discussing whether shifts in attention might provide counterexamples to representationalism. Overt shifts in attention lead to obvious changes in visual content. Moving the eyes typically changes the viewer-relative spatial properties of the items seen and brings new objects and properties into view. The real question is whether covert shifts in attention can change visual phenomenology without changing visual content. So it is clear from context that Chalmers and Nanay

\footnotetext{
${ }^{1}$ One might think that DETERMINACY-based responses to the examples of Nickel, Chalmers, and Speaks should never have seemed like strong responses. In the case of Nickel's grid, for example, the attended squares look more prominent. And prominence, it might be thought, is not itself a matter of visual determinacy. But the version of representationalism under consideration is not a thesis about the explanation of phenomenology. Rather, it is a thesis about supervenience. In order to falsify representationalism we need an example where visual phenomenology changes and yet visual content remains the same. And one way for visual content to change is for the determinacy of visual content to change. Furthermore, prominence might be a phenomenological correlate of the determinacy of representational content. When representation becomes more determinate, the subject receives more visual information about the stimulus - she can see, for example, not only that the stimulus is red but that it is specifically scarlet. And perhaps more information translates to more prominence. Note that peripheral vision is significantly lacking both in determinacy and in the prominence of the items seen.
} 
intend their comments to apply to covert shifts in attention and hence that both endorse DETERMINACY. Indeed, Nanay confirms that his views apply to covert attention in his 2011, where he notes that "[c] hange in attention is possible without moving our eyes at all" (558).

This support for DETERMINACY is understandable. After all, if I would like to know the specific color of an object, I would attend to the object, even if only covertly. If asked about the color of an object that was previously visible to me but unattended, I would likely have a relatively non-specific response. Perhaps I would only be able to say that the object was colored (as opposed to black/white/gray). Or perhaps I would be able to say that it was red, but I would likely be unable to say that it was specifically scarlet, or specifically crimson, etc. Covert attention aids property identification. A plausible explanation of this fact is that covert attention increases the determinacy of visual content. There are, however, other plausible explanations. One possibility is that attention, including covert attention, helps to provide cognitive access to what we are already visually conscious of. Indeed, Block $(1995,2007,2011)$ has advocated just this idea in another context. So perhaps there is no change in the determinacy of visual content - only a change in the availability of this information for thought and report. Ganson and I (2013: 407) suggest two additional possibilities:

Even if we grant that covert attention makes us better able to identify the (maximally) specific shade of color before us, there are a number of competing explanations of how it is that covert attention contributes to this result. One possibility is indeed that the representational content has become more determinate, but the example at hand does nothing to rule out the alternative hypothesis that covert attention improves discriminatory capacities by reducing external noise or the further alternative hypothesis that it affects our powers of discernment by improving 
processing at the decisional level.

Since there are other plausible explanations of why covert attention aids property identification, reflection on its familiar benefits does not provide much support for the idea that such attention increases the determinacy of visual content.

Ganson and I (2013) claim that DETERMINACY is on firm ground but that evidence from perceptual psychology is required. We rely on the same research as Stazicker (2011) in his defense of DETERMINACY, and we make the same mistake as Stazicker in our interpretation of this evidence. Nanay (2011) also mentions that this research supports his earlier claims about attention and visual determinacy. Each of these authors appeals to the work of Yaffa Yeshurun and Marisa Carrasco on visual spatial resolution. Visual spatial resolution is defined as the ability to see fine detail (Yeshurun and Carrasco 2008: 104; Carrasco 2011: 1500). Yeshurun and Carrasco have found that covert attention often increases spatial resolution, allowing finer details to be seen (Yeshurun and Carrasco 1998; Yeshurun and Carrasco 1999; Carrasco, William, and Yeshurun 2002; Yeshurun and Carrasco 2008; Yeshurun, Montagna, and Carrasco 2008).

Stazicker, Nanay, Ganson, and I were assuming that an increase in spatial resolution corresponds to an increase in the determinacy of visual content. Though none of us defended this assumption, the idea is fairly intuitive: if covert attention allows us to see finer details, it would seem that covert attention allows the representation of finer-grained, i.e. more determinate, properties. Hence, we interpreted the work of Yeshurun and Carrasco as evidence that covert attention sometimes affects the determinacy of visual content.

On reflection, however, there is little reason to believe that an improvement in spatial resolution 
involves a change in the determinacy of visual content. I will explain this claim by reference to the perception of Landolt squares.

A Landolt square is a square with a small gap in one side. The subject views a computer display, fixing her eyes on a point in the center of the display (the "fixation point"). A cue then appears. The cue appears either at the fixation point or in the visual periphery, where the Landolt square will soon appear. In either case the cue draws covert attention. After the cue is flashed, the Landolt square appears. (See Figure 2.) The subject's task is to indicate which side of the Landolt square contains the gap (left side vs. right side). The gap size is adjusted until performance reaches a given level of accuracy (e.g. $75 \%$ ). The task is used to measure visual spatial resolution. With good spatial resolution the task can be performed accurately even when the gap is very small. When the subject attends to the Landolt square, the gap size required for accurate localization is smaller than when the subject attends to the fixation point (Yeshurun and Carrasco 1999; Carrasco, Williams, and Yeshurun 2002). Further, the effect does not seem to be due to cue bias or some other non-perceptual mechanism. Covert attention improves visual spatial resolution.

Covert attention reduces the gap size needed for $75 \%$ accuracy by approximately $.02^{\circ}$. (This is noted by Gobell and Carrasco (2005: 649), discussing Yeshurun and Carrasco 1999 and Carrasco, Williams, and Yeshurun 2002.) This difference of $.02^{\circ}$ corresponds to a reduction in the gap size required for $75 \%$ accuracy from about $.15^{\circ}$ to about $.13^{\circ}$. (See Figure $3 \mathrm{~b}$ in Yeshurun and Carrasco 1999. Note that the figure is labeled in arc minutes rather than degrees.) Using these numbers to illustrate, we can say that an attended $.13^{\circ}$ gap results in the same accuracy as an unattended $.15^{\circ}$ gap. 


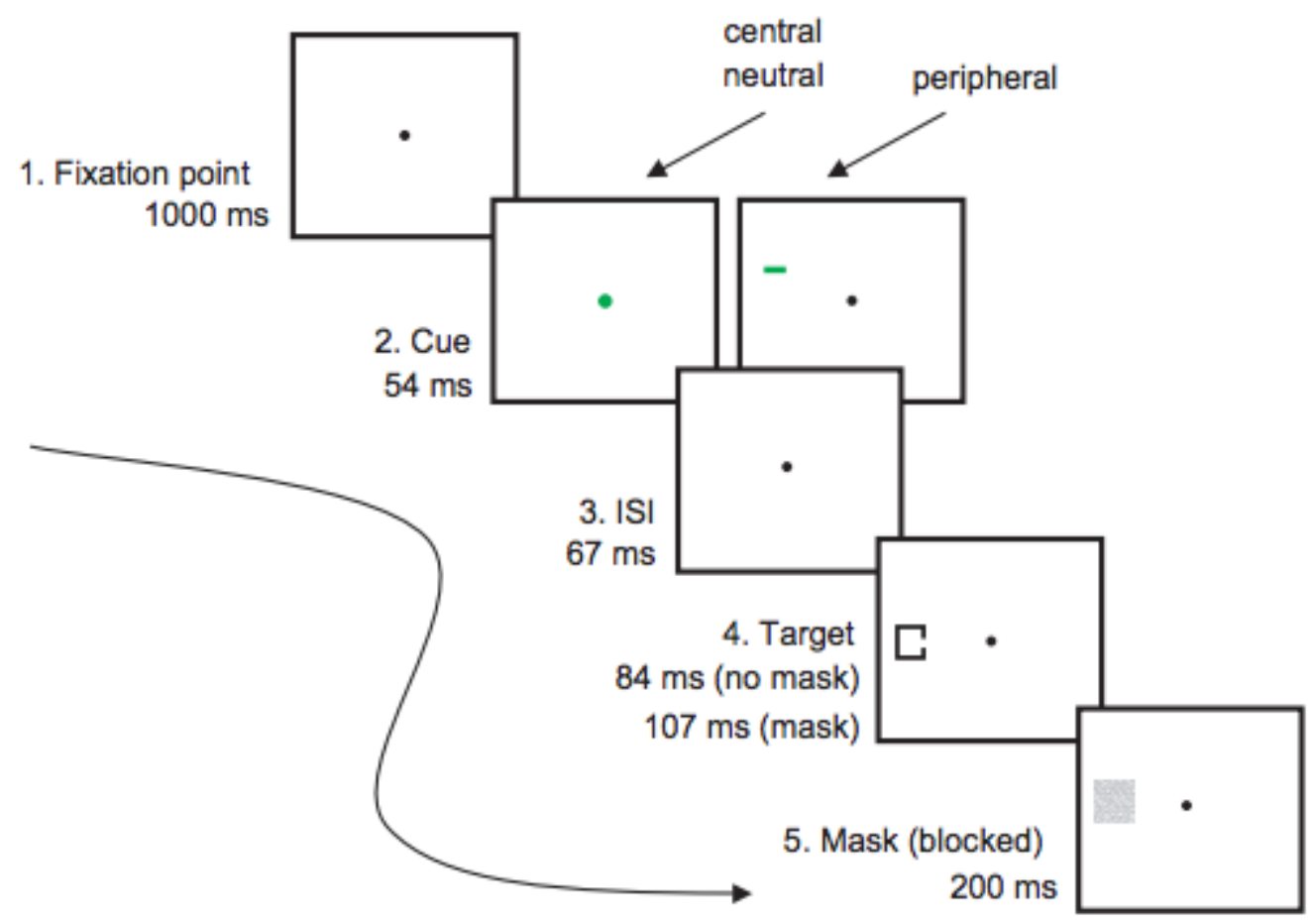

Figure 2. Each of these five steps depicts the display at a different point in time. The Landolt square is visible to the left of the fixation point in step 4. At step 3, "ISI" stands for “inter-stimulus interval." Reprinted with permission from Carrasco, Williams, and Yeshurun (2002): the Association for Research in Vision and Ophthalmology.

A plausible explanation of this fact is that an attended $.13^{\circ}$ gap results in the same visual state as an unattended $.15^{\circ}$ gap. After all, if an attended $.13^{\circ}$ gap results in the same visual state as an unattended $.15^{\circ}$ gap, then the two gaps will result in the same behavior and hence the same accuracy on the localization task. In that case, covert attention will affect visual content in the manner illustrated in Table 1. The exact numbers are not essential but are simply illustrative. What matters is that covert attention need not change the determinacy of visual content. Compare the visual content for the $.13^{\circ}$ gap with and without attention. Without attention, vision represents that the gap is between $.08^{\circ}$ and $.18^{\circ} .{ }^{2}$

\footnotetext{
${ }^{2}$ What if you do not accept the assumption that visual content is indeterminate? What if you believe conscious vision represents that the gap is exactly $.13^{\circ}$, or exactly $.14^{\circ}$, etc.? Then my work is done! I am
} 
With attention, vision represents that the gap is between $.10^{\circ}$ and $.20^{\circ}$. The property of being between $.08^{\circ}$ and $.18^{\circ}$ is not more determinate than the property of being between $.10^{\circ}$ and $.20^{\circ}$, as the former property is not more specific than the latter - both involve a range of $.10^{\circ}$. For the same reason the property of being between $.10^{\circ}$ and $.20^{\circ}$ is not more determinate than the property of being between $.08^{\circ}$ and $.18^{\circ}$. Hence, covert attention need not change the determinacy of visual content, even when covert attention improves visual spatial resolution.

Table 1

\begin{tabular}{|c|c|}
\hline Actual gap size & Visual content \\
\hline $.13^{\circ}$ (unattended) & $.08^{\circ}<$ gap size $<.18^{\circ}$ \\
\hline $.13^{\circ}$ (attended) & $.10^{\circ}<$ gap size $<.20^{\circ}$ \\
\hline $.15^{\circ}$ (unattended) & $.10^{\circ}<$ gap size $<.20^{\circ}$ \\
\hline
\end{tabular}

There are two points to keep in mind when evaluating the plausibility of the explanation I have just sketched. (1) There is independent reason to believe that an attended gap of approximately .13 results in the same visual state as an unattended gap of approximately $.15^{\circ}$. (2) For stimuli other than just Landolt squares, there is reason to believe that an attended stimulus results in the same visual state

arguing that covert attention need not change the determinacy of visual content - which would be true if visual content is never less than maximally determinate.

Note that a $.13^{\circ}$ gap is a finer (i.e. smaller) gap than a $.14^{\circ}$ gap but that the property of being $.13^{\circ}$ is not finer-grained (i.e. more determinate) than the property of being $.14^{\circ}$. Both properties are maximally determinate. 
as a larger, unattended stimulus. I will explain each of these points in turn.

(1) Gobell and Carrasco (2005) presented subjects with two Landolt squares at a time.

Subjects were told that they should respond to the Landolt square with the larger gap and report whether that gap was located in the top or the bottom of the square. Gobell and Carrasco were interested in whether subjects (i) judged the gap in the left-hand square to be larger and thus used the left hand to respond, or (ii) judged the gap in the right-hand square to be larger and thus used the right hand to respond. (See Figure 3.)

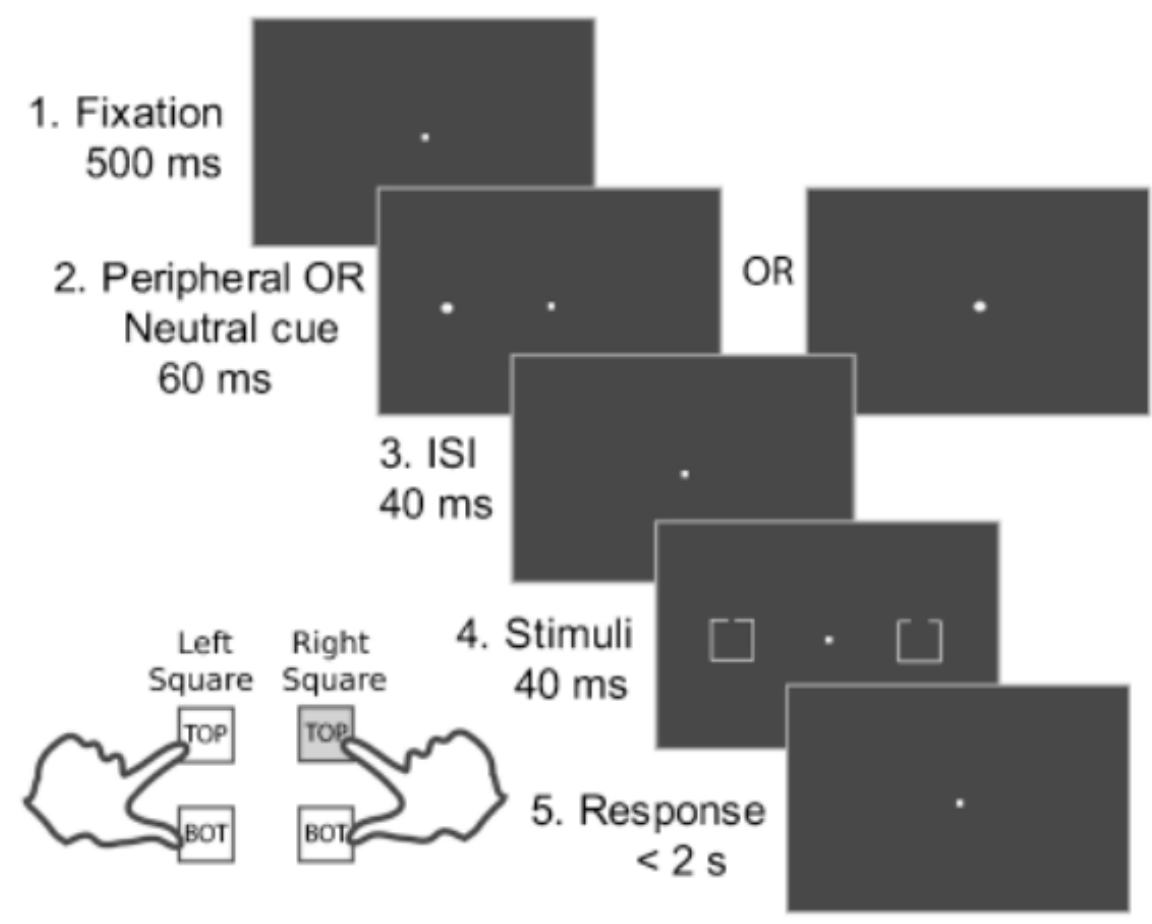

Figure 3. Reprinted with permission from Gobell and Carrasco (2005): the American Psychological Society. 
Gobell and Carrasco found that an attended $.18^{\circ}$ gap was judged to be the same size as an unattended $.20^{\circ}$ gap, and that an attended $.20^{\circ}$ gap was judged to be the same size as an unattended $.23^{\circ}$ gap. More precisely, they found that subjects were as likely to respond to an attended Landolt square containing a $.18^{\circ}$ gap as they were to respond to an unattended Landolt square containing a $.20^{\circ}$ gap, and that subjects were as likely to respond to an attended Landolt square containing a $.20^{\circ}$ gap as they were to respond to an unattended Landolt square containing a $.23^{\circ}$ gap.

Gobell and Carrasco designed the study and performed control experiments to rule out the possibility that the results were due to a non-perceptual mechanism such as cue bias. A plausible interpretation of their findings is that an attended Landolt square results in the same visual state as an unattended Landolt square with a larger gap. Indeed, that is the conclusion of Gobell and Carrasco, who say that covert attention leads to a larger "perceived" or "apparent" gap. Note that the magnitude of the effect is $.02^{\circ}$ or $.03^{\circ}$, depending on which square is attended. So there is independent reason to believe that an attended gap of approximately $.13^{\circ}$ results in the same visual state as an unattended gap of approximately $.15^{\circ}$-independent of the explanation of resolution enhancement illustrated by the paragraph preceding Table 1.

(2) Yeshurun, Carrasco, and their colleagues use a variety of stimuli to demonstrate that covert attention improves visual spatial resolution - they do not simply rely on the perception of Landolt squares. In general, covertly shifting attention to a stimulus results in performance that would otherwise be seen with a larger, unattended stimulus. ${ }^{3}$ This finding can be explained by supposing that an attended

\footnotetext{
${ }^{3}$ This is true even when spatial resolution is already too high for the task and thus covert attention impairs performance by increasing spatial resolution. Yeshurun and Carrasco (1998: 74) note that, in a test of such circumstances, covertly attending to the target shifts peak performance in a manner similar to increasing the scale of the stimuli.
} 
stimulus results in the same visual state as a larger, unattended stimulus. And indeed, for stimuli other than just Landolt squares, there is reason to believe that this supposition is true. Carrasco (2011: 1505-7) reviews a number of mechanisms that might underlie covert attention's effects on spatial resolution, at least two of which should cause an attended stimulus to result in the same visual state as a larger, unattended stimulus.

First, imagine that an optical sensor is placed on a sports field. The sensor points down the sideline to detect when players step out of bounds. Say that the sensor's "receptive field" is the portion of space within which an object will trigger the sensor. Suppose the sensor is bumped, so that it points in the wrong direction slightly - i.e. the sensor's receptive field is shifted. In that case the sensor will issue misleading reports about the players' locations.

A neuron will similarly issue misleading reports about the location of a stimulus if the neuron's receptive field is shifted and the visual system does not take the shift into account. Receptive fields shift towards the focus of covert attention. If this shift is not taken into account, then, as far as the resulting visual state is concerned, it will be as if an attended object's boundary is located at a receptive field's normal position when the boundary is actually closer to the attentional focus than that. (See Figure 4.) In other words, the resulting visual state will be as if the attended object is larger than it really is. ${ }^{4}$ Anton-Erxleben, Henrich, and Treue (2007) argue that this shifting of receptive fields can explain why covert attention increases the perceived size of moving dot patterns. It would also explain why line

\footnotetext{
${ }^{4}$ Perception of size is affected, and not simply perception of object location, because the receptors do not all shift in the same direction. A receptor to the left of the focus of attention will shift to the right, a receptor to the right of the focus of attention will shift to the left, and a receptor at the focus of attention will not shift at all. Hence, the attended object will not simply appear to be shifted to the left or right (or shifted in any other direction, for that matter). Rather, the object's boundaries will all appear to be further from the center of the object than they actually are.
} 
segments and shape contours are "repelled" from the focus of attention (Suzuki and Cavanagh 1997;

Fortenbaugh, Prinzmetal, and Robertson 2011).

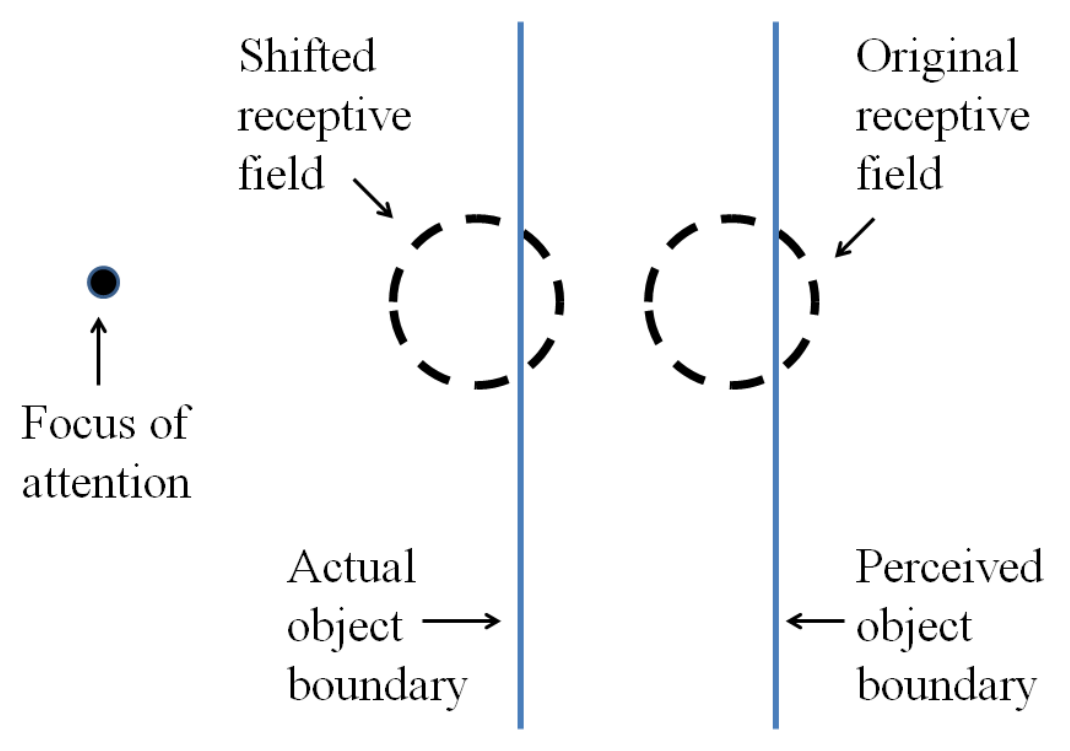

Figure 4. Receptive fields shift towards the focus of covert attention. If the visual system does not take the shift into account, then object boundaries will be "repelled" from the focus of attention, in the helpful metaphor of Suzuki and Cavanagh (1997).

Carrasco discusses a second mechanism of resolution enhancement that should cause an attended stimulus to result in the same visual state as a larger, unattended stimulus. Imagine that you measure the size of various objects by judging how fully they fill a given bowl. If your bowl shrinks and you do not take the shrinking into account, then you will think that a given object is larger than it really is. Similarly, if a neuron's receptive field shrinks but the visual system does not take account of the shrinking, then as far as the resulting visual state is concerned it will be as if a larger stimulus is present. Covert attention shrinks receptive fields at the attended location. If that shrinking is not taken into 
account, then the visual state resulting from an attended stimulus will mimic the visual state that would result from a larger, unattended stimulus.

The upshot is this. When covert attention enhances spatial resolution, it might be that there is no change in the determinacy of visual content. The resulting visual state might simply match the state that would result from a larger, unattended stimulus. This possibility gains plausibility because covert attention seems to increase the visual representation of size (see (2) above) and, at least for Landolt squares, the magnitude of this effect is just about right (see (1) above).

Spatial resolution can be operationally defined by reference to the maximum detectable spatial frequency, i.e. the maximum detectable "rate of change of a phenomenon across space" (Stazicker 2011: 172). While I have concentrated on the perception of size, parallel remarks apply to the perception of spatial frequency. Indeed, here is Gobell and Carrasco describing covert attention's effect on the perception of spatial frequency: "The pattern [of neural activation] under the influence of attention will be consistent with a pattern that would be observed in the absence of attention if the stimulus were of a higher spatial frequency. This would result in the phenomenological experience of a higher spatial frequency" (2005: 650). And experiencing a stimulus as possessing a higher spatial frequency need not involve a change in the determinacy of the properties attributed by vision to the stimulus. For example, the property of being between 50 and $70 \mathrm{cpd}$ (cycles per degree) is not more determinate than the property of being between 40 and $60 \mathrm{cpd}$. The two properties have the same degree of specificity, as they each involve a range of $20 \mathrm{cpd}$. In general, the visual state resulting from an attended stimulus might simply match the state resulting from an unattended stimulus that is larger, higher in spatial frequency, 
etc., with no change in the determinacy of visual content. ${ }^{5}$

Some of the evidence reviewed above regards involuntary covert attention - attention that is not purposefully shifted but is captured by the sudden appearance of a cue carrying no useful information. Both involuntary and voluntary covert attention repel line segments (Suzuki and Cavanagh 1997) and shift and shrink receptive fields (Carrasco 2011: 1505f), and this fact suggests that even voluntary covert attention should increase the representation of stimulus size. However, the results of Gobell and Carrasco 2005 pertain to involuntary covert attention, which often has different effects from its voluntary counterpart (as briefly reviewed by Yeshurun, Montagna, and Carrasco 2008: 92; see also Prinzmetal and Landau 2010). Might voluntary covert attention sometimes increase the determinacy of visual content?

It is indeed possible that voluntary covert attention sometimes increases visual determinacy. It is even possible that involuntary covert attention sometimes increases visual determinacy. I have not attempted to rule out these possibilities. I have simply argued that there is a very plausible explanation for covert attention's effect on spatial resolution that does not involve changes in the determinacy of visual content. As a result, Yeshurun and Carrasco’s work on spatial resolution does not confirm DETERMINACY.

I would go a bit further, however, and claim that explaining Yeshurun and Carrasco's results by

\footnotetext{
${ }^{5}$ Further down on p. 650, Gobell and Carrasco write: "Attention increases spatial resolution ... providing a more precise estimate of the location of the ends of the line segments." This might sound like talk of an increase in the determinacy with which the line segments are represented. However, the authors continue: "This diminishes the estimation errors produced without attention, thereby producing the appearance of a larger gap" (emphasis added). So Gobell and Carrasco are simply describing a mechanism whereby a smaller Landolt gap produces the experience of a larger gap. The effect they posit of covert attention on visual experience is simply an increase in the perception of gap size, not an increase in determinacy.
} 
reference to a change in perceived size is a better explanation than explaining these results by reference to a change in the determinacy of visual content. As explained in (1) and (2) above, we have independent evidence that covert attention affects the perception of size. It is more parsimonious to explain Yeshurun and Carrasco's spatial resolution results by this known effect than by positing a new effect of covert attention - namely, an effect on visual determinacy - for which we do not have independent experimental evidence.

For the sake of completeness, I should note that there is a plausible argument in support of DETERMINACY. Muscles internal to the eye can alter the shape of the lens, allowing the world to remain in focus as the eyes fixate items at various distances from the viewer. This accommodation of the lens is often accompanied by eye movement and, when it is, we are dealing with overt attention. If you try to bring an object into focus that is closer to you than the previously fixated point, for example, your eyes will naturally rotate inward to fixate the object. However, we can imagine dissociating accommodation of the lens from eye movement. Suppose you hold your eyes fixed on a given point and vary the distance at which you are attending, resulting in accommodation of the lens with no eye movement. Doing so will change which items look blurry and how blurry they look. Items will look more blurry the farther they are from the attended point. Perhaps blurry vision is a matter of indeterminate visual content (Tye 2002; but see Allen 2013 for a dissenting view). If so, then covert shifts in attention will affect visual determinacy when they result in accommodation of the lens. ${ }^{6}$

\footnotetext{
${ }^{6}$ Ganson and I (2013) used a definition of covert attention that precludes this scenario. We defined covert attention as involving no muscular contraction, whereas the present paper defines it as involving no movement of the eyes. The former definition excludes focusing of the eye while the latter does not. In the psychological literature, covert attention is usually characterized by reference to eye movement rather than muscular contraction in general (e.g. Gobell and Carrasco 2005: 644; Carrasco 2011: 1486).
} 
This result, while interesting, will not save the relevant version of DETERMINACY. Technically, DETERMINACY should be defined as the claim that covert shifts in visual attention sometimes affect the determinacy of non-blurry vision. The examples of Nickel, Chalmers, and Speaks each involve a significant change in phenomenology with no noticeable change in the blurriness of the stimuli. Indeed, in these examples the viewer is not shifting attention between near and far items, so there is little reason to expect accommodation of the lens. And none of the advocates of DETERMINACY mention anything about blurry vision - they are talking about covert attention changing the determinacy of non-blurry vision. So, on the relevant version of DETERMINACY, which is also the version endorsed by previous philosophers, we have little reason to believe that the claim is true. ${ }^{7}$

I close by considering the implications of the present paper for representationalism. The examples of Nickel, Chalmers, and Speaks involve changes in phenomenology with no obvious changes in visual content. One possibility is that these examples involve covert shifts in attention that change the determinacy of visual content. While this is indeed a possibility, previous authors are mistaken that there is good evidence covert attention sometimes has such an effect. Hence, this response to the examples of Nickel, Chalmers, and Speaks is not a strong one.

My discussion of DETERMINACY makes salient an alternative response to the examples of Nickel, Chalmers, and Speaks - namely, that these examples involve changes in the representation of size.

\footnotetext{
${ }^{7}$ Wayne $\mathrm{Wu}$ has suggested to me that Carrasco takes the work of Yehoshua Tsal to support DETERMINACY. Tsal suggests that attending to a location shrinks an "attentional receptive field" (ARF) at that location, "a hypothetical construct that operates as a functional receptive field" (Carrasco 2011: 1505). Note, however, that in Figure 14 of Carrasco 2011 (p. 1506) the effect of shrinking ARFs is to (i) decrease perceived line length, and (ii) increase perceived gap size (from zero to some positive amount). As should be clear by now, such effects on perceived size are not in themselves effects on the determinacy of visual content. See the left-hand columns of p. 1505 and p. 1506 (ibid.) for references and brief discussion of how ARFs are hypothesized to function.
} 
Recall my suggestion that an attended object results in the same visual state as a larger, unattended object. However, I have argued only that covert attention is plausibly affecting the representation of size in the experiments interpreted as supporting DETERMINACY. Nickel, for one, explicitly rejects the suggestion that in his example attention affects the perception of size (288). More generally, it is well known that covert attention can affect the perception not only of size but also of other properties such as saturation (Fuller and Carrasco 2006) and luminance contrast (Carrasco, Ling, and Read 2004; Liu, Abrams, and Carrasco 2009). Yet these well-known facts have not deterred opponents of representationalism, in large part because it is far from obvious that the relevant experimental results extend to all possible experiences. Whether, in the examples at hand, covert attention is affecting the representation of properties such as size, saturation, and contrast is a matter that I leave open.

\section{Acknowledgments}

Thanks to Wayne Wu and two anonymous reviewers at Philosophical Psychology for helpful comments. I also thank two anonymous reviewers at the Southern Journal of Philosophy for comments on a previous version of this paper. Special thanks are due to one reviewer from the latter journal who provided a PDF of extensive and invaluable comments, which greatly improved the paper. The most special thanks are reserved, however, for Todd Ganson, who generously read and commented on many versions of this and related papers.

\section{References}

Allen K. (2013). Blur. Philosophical Studies 162 (2): 257-273.

Anton-Erxleben K., Henrich C. \& Treue S. (2007). Attention changes perceived size of moving visual patterns. Journal of Vision 7 (11): 5, 1-9.

Block N. (1995). On a confusion about a function of consciousness. Behavioral and Brain Sciences 18 (2): 227-247. 
Block N. (2007). Consciousness, accessibility, and the mesh between psychology and neuroscience. Behavioral and Brain Sciences 30 (5-6): 481-499.

Block N. (2011). Perceptual consciousness overflows cognitive access. Trends in Cognitive Sciences 15 (12): 567-575.

Byrne A. (2001). Intentionalism defended. Philosophical Review 110 (2): 199-240.

Carrasco M., Williams P.E. \& Yeshurun Y. (2002). Covert attention increases spatial resolution with or without masks: Support for signal enhancement. Journal of Vision 2: 467-479, DOI 10:1167/2.6.4.

Carrasco M., Ling S. \& Read S. (2004). Attention alters appearance. Nature Neuroscience 7: 308-313.

Carrasco M. (2011). Visual attention: The past 25 years. Vision Research 51: 1484-1525.

Chalmers D. (2004). The representational character of experience. In B. Leiter (ed.), The Future for Philosophy (pp. 153-181). Oxford: Oxford University Press.

Dretske F. (1995). Naturalizing the Mind. Cambridge, MA: MIT Press.

Fortenbaugh F.C., Prinzmetal W. \& Robertson L.C. (2011). Rapid changes in visual-spatial attention distort object shape. Psychological Bulletin Review 18: 287-294.

Fuller S. \& Carrasco M. (2006). Exogenous attention and color perception: Performance and appearance of saturation and hue. Vision Research 46: 4032-4047.

Ganson T. \& Bronner B. (2013). Visual prominence and representationalism. Philosophical Studies 164 (2): 405-418.

Gobell J. \& Carrasco M. (2005). Attention alters the appearance of spatial frequency and gap size. Psychological Science 16 (8): 644-651.

Harman G. (1990). The intrinsic quality of experience. Philosophical Perspectives 4: 31-52.

Liu T., Abrams J. \& Carrasco M. (2009). Voluntary attention enhances contrast appearance. Psychological Science 20 (3): 354-362. 
Nanay B. (2010). Attention and perceptual content. Analysis 70: 263-270.

Nanay B. (2011). Ambiguous figures, attention, and perceptual content: Reply to Jagnow. Phenomenology and the Cognitive Sciences 10 (4): 557-561.

Nickel B. (2007). Against intentionalism. Philosophical Studies 136: 279-304.

Prinzmetal W. \& Landau A.N. (2010). Dissecting spatial visual attention. In V. Coltheart (ed.), Tutorials in Visual Cognition (pp. 43-66). Hove: Psychology Press.

Speaks J. (2010). Attention and intentionalism. Philosophical Quarterly 60 (239): 325-342.

Stazicker J. (2011). Attention, visual consciousness and indeterminacy. Mind \& Language 26 (2): 156-184.

Suzuki S. \& Cavanagh P. (1997). Focused attention distorts visual space: An attentional repulsion effect. Journal of Experimental Psychology: Human Perception and Performance 23 (2): 443-463.

Tye M. (2002). Representationalism and the transparency of experience. Nous 36 (1): 137-151.

Yeshurun Y. \& Carrasco M. (1998). Attention improves or impairs visual performance by enhancing spatial resolution. Nature 396 (6706): 72-75.

Yeshurun Y. \& Carrasco M. (1999). Spatial attention improves performance in spatial resolution tasks. Vision Research 39: 293-306.

Yeshurun Y. \& Carrasco M. (2008). The effects of transient attention on spatial resolution and the size of the attentional cue. Perception \& Psychophysics 70 (1): 104-113.

Yeshurun Y., Montagna B. \& Carrasco M. (2008). On the flexibility of sustained attention and its effects on a texture segmentation task. Vision Research 48: 80-95. 